\title{
Critical Mass and Discontinued Use of Social Media
}

1. Thomas Chesney ${ }^{1, *}$ and

2. Shaun Lawson ${ }^{2}$

Article first published online: 19 NOV 2013

DOI: 10.1002/sres.2231

Copyright (C) 2013 John Wiley \& Sons, Ltd.

Issue

Systems Research and Behavioral Science

Volume 32, Issue 3, pages 376-387, May/June 2015

Keywords:

- technology fad;

- simulation;

- $\quad$ social network

\section{Abstract}

Using simulation, this study compares a critical mass of adopters with a critical mass of those who discontinue their adoption of social media. A network of reflex agents is simulated where each agent has an unchanging threshold and will adopt social media if the number of their friends who have adopted is greater than it. In the first study, the size of the critical mass that adopts is varied, and in the second, the size of the critical mass that discontinues use is varied. The studies show that a critical mass of leavers can cause a community to fail and that this mass can potentially be as small as that needed to influence a community to succeed; although given a certain critical mass, their leaving is less likely to cause failure than their adoption is success. This influence of the critical mass is facilitated by network structure. Copyright @ 2013 John Wiley \& Sons, Ltd

\section{Critical Mass and Technology Adoption}

In a social network, the position of each actor can alone influence others in the network (Tang, 2011). In this paper, we model and explore using simulation, the effect of users joining and leaving a social network, to understand how a critical mass affects both the success and failure of a social media platform. Social networks are of increasing importance to social science (Willer, 2007; Centola, 2009; Roca et al., 2012) and to electronic commerce research in particular (Cortizo et al., 2011; Liang and Turban, 2011) as services such as Facebook and Twitter have opened avenues for new business models (Liang et al., 2011) something referred to as social commerce (Stephen and Toubia, 2010). According to a recent special issue on the topic, social commerce raises a variety of new questions for e-commerce researchers and the field could become one of the most challenging research arenas in the coming decade (Liang and Turban, 2011).

Social commerce is powerful because of the speed and distance that information spreads through social networks, which can generate considerable economic value (Stephen and Toubia, 2010) (for example, Amblee and Bui, 2011 on the effect of 'electronic word of mouth' marketing). The adoption of social networking technology then becomes an important research question. Research into technology adoption is well established 
(Benbasat and Barki, 2007) but research into why users discontinue their use of technology is also critical, and work here has begun (Prins et al., 2009). In fact, users' postadoption behavior has now emerged as a key information systems research theme (Venkatesh et al., 2011) and an interesting subtheme of this work is why once successful technologies fail, which is where this paper makes its contribution.

Discontinuation of use is a real threat for social media service providers, and by extension, it is also a risk for commercial organisations that wish to build business models on social networks. Recent developments in online social media have seen some services' popularity-in terms of numbers of users-wane whilst others continue to gain new users. The online social network Facebook remains the dominant online social media service in English speaking countries having overtaken services such as MySpace in terms of everyday usage and uptake. Newer services such as Google Buzz and Google+ attempted—but failed—to take market share away from Facebook.

For several decades, researchers have been interested in the question of why people adopt information technologies (for instance, Hirschheim,2007). One prominent part of this work is critical mass theory (Marwell and Oliver, 1993). A critical mass is a small group of early adopters who are highly motivated to use the technology. Critical mass theory provides one explanation of how technologies 'catch on' where the critical mass influences other potential users to adopt. The typical adoption curve is shown in Figure 1. The curve starts as the critical mass slowly influences others until a tipping point is reached, after which rapid adoption occurs until a plateau where there are few new adopters (Markus, 1987).

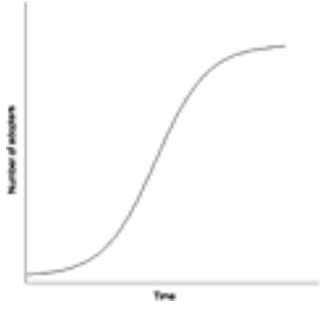

Figure 1. The S-curve of technology adoption

Critical mass theory also suggests a potential explanation of why initially successful technologies eventually fail: a critical mass stops using the technology and influence others to do the same.

Social media services such as Facebook, Twitter and Yammer rely on word of mouth marketing. In other words, they rely on a network of individuals where small groups of users (the critical mass) adopt and influence others to follow suit, and these new adopters influence others and so on. The common phrase to describe this is that the phenomenon has gone viral. For example, the popular microblogging service Twitter only used word of mouth marketing, encouraging it with the strategic positioning of public Twitter feeds where potential early adopters might have seen them (Levy, 2007). Social media services allow friends to connect with each other. The services are meaningless without these connections. This makes a user's continued use dependent on the continued use of others.

The current study makes use of both of these facts-the necessity of connections to facilitate uptake and the necessity of connections to facilitate continued use-to simulate data to model how early adopters affect take-up across the community of potential users and how the same community-once successful—is affected by users leaving. 


\section{Influence and Online Social Networks}

Following (Granovetter, 1978) who-drawing on the work of (Schelling, 1971) —uses a threshold to illustrate how two almost identical crowds can arrive at vastly different group behavior, influence is often modelled by a threshold rule (Watts and Dodds, 2007; Centola, 2010). Watts and Dodds (2007) describe a threshold rule as one where 'individuals will switch from $A$ to $B$ only when sufficiently many others have adopted B in order for the perceived benefits of adopting a new innovation to outweigh the perceived cost'. In the language of social media, the probability,p(adopt), of a user $i$ adopting a service is 1 whenever the number of their friends $m$ (their network neighbours) who have adopted is greater than their threshold $\phi$ :

$p($ adopt $)= \begin{cases}1 & \text { if } m_{i}>\phi_{i} \\ 0 & \text { if } m_{i} \leq \phi_{i}\end{cases}$

Easley and Kleinberg (2010, p.500) give a good summary of how a threshold rule arises by considering a coordination game where individuals must choose between two choices $A$ and $B$ where an incentive exists for any two connected individuals to match their choice. If two connected individuals both choose $A$, they obtain a benefit or payoff of $a$. If both adopt $\mathrm{B}$, they obtain a payoff of $b$, and if their choices do not match, they obtain a pay-off of 0 . To see how the threshold arises, consider what one of them should do to maximise their payoff if some of their neighbours adopt $\mathrm{A}$ and some adopt $\mathrm{B}$. If a fraction, $p$ of $d$ neighbours adopt $\mathrm{A}$ then $(1-p) d$ adopt B. If an individual chooses $\mathrm{A}$, they obtain a pay-off of $p d a$, and $(1-p) d b$ if they choose $\mathrm{B}$. $\mathrm{A}$ is therefore the better choice when

$p \geq \frac{b}{a+b}$

This is the threshold rule: if $b /(a+b)$ of an individual's neighbours choose A, then they should too.

In a review of technology adoption literature, Poe (2011) discusses how new communication technologies both arise and are adopted. Focusing on the work of Harold Innis (Innis, 1950; Innis, 1951), Poe argues that communication technologies are pulled into our lives, and he distills how this happens into seven rules. One of these rules is that only organised interests can produce the demand necessary to pull a new technology into mass use. Organised interests overcome adoption barriers. Such a barrier exists for the first person to adopt a new communication technology. For instance, if only one person owns a telephone, it is useless to them, but if two people organise themselves to each own a phone, then they can now be used to talk to each other. As every new person adopts, the usefulness of the device increases for everyone. Thus, the ultimate success of a communication technology is dependent on a small group of early adopters, the critical mass. One way it is believed that a critical mass pulls a new technology into adoption is by social influence.

Social influence appears as a construct in many theories of technology adoption although often different terminology is used to label it. The term social influence is the one used in the Unified Theory of Acceptance and Use of Technology model (Venkatesh et al., 2003), but the same construct has appeared as 'subject norm' in the theory of reasoned action (Ajzen and Fishbein, 1973) and the theory of planned behavior (Ajzen, 1991), as 'social factors' in the model of PC utilisation (Thompson et al., 1991), and as 'image' in innovation diffusion theory (Rogers,1995). Each of these constructs measures how much an individual perceives their behavior is influenced 
by the way in which they believe others will view them as a result of using the technology. In the Unified Theory of Acceptance and Use of Technology model, social influence is measured by the following items, which users indicate agreement with on a Likert scale:

1. People who influence my behavior think that I should use the system.

2. People who are important to me think that I should use the system.

3. The senior management of this business has been helpful in the use of the system.

4. In general, the organisation has supported the use of the system.

Under the threshold rule social influence 'builds up' with each friend who has adopted adding to it until the threshold is breached. A threshold rule has proven a convenient and realistic tool for modelling human behavior (Schelling, 1973; Valente, 1996; Morris, 2000), and in a recent study, Centola, (2011) found that a threshold rule is used by members of online social networks in their decision to adopt certain behaviors.

\section{Network Simulation}

Adoption/discontinued use is examined here by simulation. Social science has been slow to adopt simulation as a research method (Bainbridge,2010). Philosophers of science have started to consider it, with (Winsberg, 2010) giving a review, devoting much of the book to examining the question of where simulation sits between theorising and experimenting. Although certainly not a position beyond criticism, the current work views its simulations as objects that are experimentally manipulated, with the computer being a physical stand in for a community of microbloggers. An alternative way of examining the current research question would be to incentivise a number of human subjects to form a Twitter-like microblogging community and manipulate a critical mass of them to start sending posts. Although many might argue that a fundamental difference exists between these two methods, both in fact are simulations (Winsberg, 2010). Following the arguments of, for example, Simon (1969) and Guala (2002), it might be claimed that the difference is that the latter shares a deep, material similarity with the phenomena of interest (they both involve humans making decisions), whereas the former does not. However, even this position is not beyond criticism with some concluding from it that it makes the distinction between simulation and experiment even more unclear by forcing a decision on when a difference becomes deep and material (Winsberg, 2010).

We justify the use of a computer being a physical stand in for an online community by our desire to have a community with identical and known social network structure and identical and well-understood decision rules, all of which can be presented with a variable critical mass of adopters and leavers. The simulated communities are populated by agents whose network structure and decision rules are based on theory. By manipulating the number of early adopters or leavers, how a community performs over a period can be measured. The first of the two elements of the model, network structure, is now examined. The second, the decision rule, is described in the next section.

Social networks (including online social networks and in fact many other types of network) are characterised by highly connected clusters within a population, with short paths between any two randomly selected people (Watts and Strogatz, 1998). This fact was famously demonstrated by Milgram's six degrees of separation experiment 
(Milgram, 1967). Imagine two groups of friends on opposite sides of the planet. If two of them to meet on a plane then suddenly there is at most three jumps between any two of them ( $A$ knows $B$, B met $C$ on the plane and $C$ knows D). This is known as the small world phenomena. For instance, Twitter and Facebook are known to be a small world network (Java et al., 2007; BBC, 2011).

The algorithm used in this study for creating a small world network was presented by Watts and Strogatz (1998) and described in detail by Watts (1999). It results in a community that has high clustering and short path lengths (Newman, 2010). Clustering can be informally explained as 'the friend of my friend is also my friend' and can be defined as: if node $A$ is related to node $B$ and $B$ is related to $C$, then-if the network exhibits high clustering-it follows that $A$ tends to also be related to $C$. Path length is the number of edges, or relationships, between any two nodes in the network and in a social network is a measure of the shortest number of acquaintances needed to link two people (refer to Table 1 for examples).

Network

Film actors

Coauthorship in mathematics

Facebook

Company directors
Path length

3.65 Watts and Strogatz, $\underline{1998}$

$\approx 4.7 \quad$ Newman, 2001

$4.74 \quad$ BBC, 2011

$\approx 3.5 \quad$ Davis et al., 2003

Table 1. Average path lengths of several social networks with sources

The small world model does not capture all characteristics of real social networks (for instance, preferential attachment Barabási and Albert, 1999), but it does give a stable social network structure that provides a platform for robust experimentation and is well supported in the literature (for example, Opuszko and Ruhland, 2013). It also demonstrates the strength of weak ties.

Strong ties (Granovetter, 1973) refer to close acquaintances, those that are close to us physically and/or emotionally. Conversely, a weak tie is a relationship with someone we exchange with rarely. The Watts/Strogatz model shows that a few weak ties between strongly tied groups drastically changes the dynamics of a network and gives short path length between any two members in it.

Centola and Macy (2007) examine the implications of this fact in a comparison of simple and complex contagions. A simple contagion exists when contact with one carrier is enough to pass it on. This could be an infectious disease but applies equally to information. Complex contagions require reinforcement, that is, contact with more than one carrier. Social influence is an example: for instance, belief in a myth probably requires more than one person to retell a story before it becomes convincing. Centola and Macy (2007) find that while a small world network structure allows the spread of simple contagions, this cannot be generalised to collective behaviors. This is because a single weak tie allows flow from one strongly tied group to another, but for complex contagion, what is required is multiple signals from one strongly tied group to another. A comparison of this work with the current study is offered in the Discussion Section.

\section{Procedure}


All-machine simulations (Jaccard and Jacoby, 2010) of two social network structures were developed in the statistical programming language $\mathrm{R}$. Each node in the networks was created to be a reflex agent (Bermudez, 2010) that would act according to a decision rule and react to the actions of other agents. The decision rule was the threshold rule described earlier: each agent had an unchanging threshold and would adopt social media if the number of their friends (network neighbours) who had adopted is greater than it. The networks were set up using the igraph package (Csárdi and Nepusz, 2006) with visualisation routines for the network images in this paper taken from the network package (Butts et al., 2007).

The first network was called fnet, for follower network. The fnet was created using the watts.strogatz.game function in igraph, which is designed to simulate a small world according to the (Watts and Strogatz, 1998) procedure which can be summarised as follows. A set number of agents are conceptually arranged in a circle and linked to their $x$ nearest neighbours, where $x$ is a variable parameter. The links are then rewired randomly. What this means is that an existing link is removed (between agent $A$ and $B$ say) and replaced with a link to another randomly chosen agent (so now, agent $\mathrm{A}$ is linked to agent $\mathrm{K}$ ). The probability of this happening for each link is another variable parameter.

The following parameters (included in full to allow replication) were used with the watts.strogatz.game function and were set after preliminary experimentation:

- The dimension of the starting lattice $=1$ (as the network records only one type of connection intended to represent Twitter's following feature where A can follow B but B does not necessarily-although could_follow $A$ ).

- The number of agents $=200$.

- The neighbourhood within which the agents will be connected $=5$.

- $\quad$ the rewiring probability $=0.1$.

- Multiple edges and loops (Newman, 2010) were not allowed.

The value for the rewiring probability was taken from Bruggeman (2008) who states that a probability of approximating this value gives short path lengths and high local clustering similarity to a real social network. The removal of multiple edges and loops means that agent $A$ and $B$ can only be connected once in the same direction (multiple edge), and A cannot be connected to itself (loop).

As hinted in the preceding description, the simulated networks were directed, which means that links between agents run from one agent to the other and this direction has meaning. This can be nicely illustrated by considering the two most popular social networking services Facebook and Twitter. Relationships on Facebook are undirected: if person $A$ is friends with person $B$, then person $B$ is friends with person $A$. On Twitter relationships are directed: person $A$ can follow person $B$ without person $B$ following person $A$.

The second network was a configuration model network that was created as a control, as suggested by Newman and Park (2003). A configuration model is a network that has a given degree sequence but in all other respects is random (Newman, 2010). This means that the number of agents each agent is connected to is specified, but those agents are chosen randomly. The number of agents in the configuration model was 200 , and the degree sequence was identical to that of fnet, with—as before-the removal of multiple edges and loops. (This was achieved using the igraph degree.sqeuence.game and simplify functions.) The result is that agents in the 
configuration model had the same number of followers as those in fnet. Both networks are shown in Figure $\underline{2}$. The clustering coefficients of fnet (0.680) and the configuration model $(0.087)$ were in line with what would be expected (Newman and Park, 2003) giving confidence that the models are created as intended. ${ }^{1}$

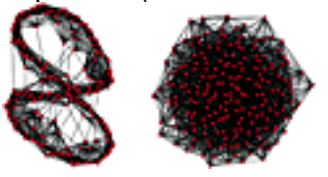

Figure 2. The fnet (left) and the configuration model (right)

Each agent was assigned a threshold. The values were normally distributed with parameters taken from a normal distribution, $N(4,1)$, and assigned randomly to agents. An identical set of threshold values was used for both communities (the fnet and the configuration model). How a threshold rule was derived was shown earlier in the Influence and Online Social Networks Section. Lacking empirical data for setting the payoffs (which were also described in the Influence and Online Social Networks Section), using a normal distribution of threshold values seems appropriate intuitively, and the parameters used were chosen after experimentation. It should be noted however that our conclusions are independent of these threshold values, and we would not expect our observations to change had alternative thresholds been used.

The following procedure was then performed on both communities: a random $x$ agent was chosen as the critical mass and forced to adopt and signal this adoption to each of their network neighbours. The entire community of agents then responded to this according to their decision rule. This adopting and responding lasted for 40 discrete periods. The value of $x$ was varied from 1 to 40 , and for each, the procedure was repeated 50 times. Then, to simulate a successful community from which a critical mass would leave, all agents were forced to adopt. A random $x$ agent was made to discontinue use, then other agents reacted to this according to their decision rule. As before titx was varied from 1 to 40 , and for each value of $x$, the procedure was repeated 50 times.

The choice of running the simulation for 40 periods was taken after experimentation as this was found to be enough to determine whether the community would succeed or fail.

\section{The 'Outsiders'}

Given the procedure used to set up the community of agents, some agents (termed 'outsiders') by chance had a higher threshold than number of people they are following, and therefore, they would never adopt in the adoption study and would leave the community immediately in the discontinued use study. The fnet had four such agents; the configuration model had 43. These outsiders put a limit on how successful each community could ever be. Although it is important to know this in the adoption study, it is not a concern as they would not artificially inflate the adoption figures. However, it is a concern in the discontinued use simulation as these agents greatly increase the critical mass of leavers. To deal with this, it was decided that because such an agent could never adopt in the adoption study, then they would be made to never leave in the discontinued use study, unless randomly chosen as part of the critical mass of leavers. This was achieved by manipulating the threshold values so the outsiders would always post- -the decision rule did not change, but their threshold value was not randomly assigned. 
The implication of doing this is a change in the size of the community. The fnet went from being a community of 200 to being one of 196 . The configuration model went to a community of 157 . In interpreting our results, we have been careful to ensure that-as with other parameter values used-what we are concluding is independent of the outsiders. The way we ensured this was by not directly comparing the absolute values of the number of adopters in fnet and the configuration model. We compared fnet in the adoption study with fnet in the discontinued use study and compared the configuration model in the adoption study with the configuration model in the discontinued use study. However, we only compared fnet with the configuration model by comparing the likelihood of success or failure in each and not the absolute results obtained in each.

\section{Defining Success}

For the analysis of results, it is necessary to define what a successful community looks like in terms of the number of adopters. The decision on this is arbitrary, and $80 \%$ was chosen as the success/failure cut-off point. Therefore, if a community achieves an adoption figure of $80 \%$ of its community of 200 minus the outsiders, then it is considered a success, otherwise it is considered a failure. Note again that although to be a success, the configuration model has to achieve fewer posting agents than fnet; the conclusions drawn in the Discussion Section are independent of this fact.

\section{Results}

The pattern of number of adopters across the 40 periods for one of the trials is shown in Figure $\underline{3}$, which has the familiar adoption S-curve (Jackson, 2008). Our results are then summarised in Figures $\underline{4}$ and $\underline{5}$. Figure $\underline{4}$ shows the maximum number of adopters across all 50 runs of the simulation and the mean number of adopters achieved in the 50 runs for both fnet and the configuration model. Figure $\underline{5}$ shows the same for leavers. Three elements of the graphs should be noted. The first is the point at which the fnet line flattens out in each of the two upper panels. These panels show the maximum number of adopters/leavers and represent what is possible rather than what is likely. The point at which the line flattens is remarkably similar in each line. This suggests that a similar size of critical mass to one that can make a community successful can also make a community fail. In fact, the minimum size of a critical mass of adopters that was found to produce a successful community was seven, whereas the minimum critical mass of leavers that was found to destroy a successful community was six.

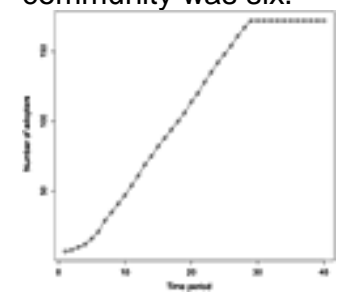

Figure 3. An example of a successful adoption in fnet with $\mathbf{x}=\mathbf{7}$ 


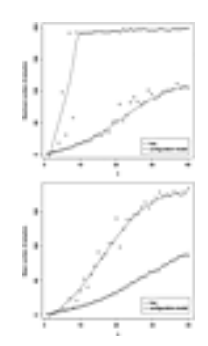

Figure 4. Maximum and mean number of adopters

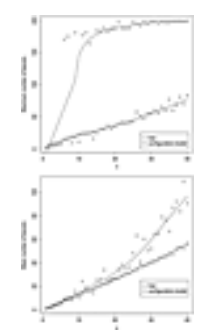

Figure 5. Maximum and mean numbers of leavers

The second element to note is the point at which the fnet lines flatten in the two lower panels. This is the mean number of adopters/leavers and represents what is likely, not what is possible. For adoption (Figure $\underline{4}$ ), the line starts to flatten at a critical mass of about 30 (the value on the $x$-axis). The point is not even visible on Figure $\underline{5}$ but is certainly greater than 40 . This suggests that the likelihood of a given critical mass influencing successful adoption is greater than the likelihood of the same critical mass causing a successful community to fail. In fact, when xreached a value of seven, it created successful communities in four out of the 50 attempts. Only one of the successful communities was destroyed by a similar-sized critical mass. When $x$ reached around 25 , successful communities were more common than failed ones. A similar pattern was seen in those communities that failed: after a certain point, failed communities were more common than successful ones, although the probability of failure was lower than the probability of success.

The third element to note is the difference between the fnet and configuration model line: in all panels, the configuration model lies to the right, meaning that adoption/failure is more likely in fnet than the configuration model and that a larger critical mass is required in the configuration model to influence success or failure. This suggests that the reason a small critical mass can have a powerful impact on the community either towards success or failure lies in the structure of the network.

\section{Discussion}

Although interesting to see how a community can be affected by a minority of people (just six or seven), this is not a key finding as these values stem from the choice of threshold values: higher threshold values would have increased the minimum size of the critical mass. The valid finding is that in a community of agents whose decisions are dependent on the decisions of others, as soon as the critical mass is able to influence at least one agent, then there is a probability that the community will succeed or fail.

We offer two conclusions. Firstly, a critical mass of leavers can cause an online community to fail. Indeed, this mass can potentially be as small as the mass needed to influence a community to succeed; although given a 
certain critical mass, their leaving is less likely to cause failure than their adopting will cause success. Secondly, the likelihood of success/failure is determined by the network structure.

The first conclusion is based on a comparison of fnet in the two studies, which held network structure, the agents' threshold values and the decision rule constant. The second conclusion is based on a relative comparison of fnet with the configuration model. Here, the difference is the network structure, with all else held constant. The structure in a social network not only makes adoption more likely but also makes it susceptible to discontinued use.

The 'contagion' modelled in this paper is complex in that a threshold must be breached for an individual to adopt. Centola and Macy (2007) find that a small world network structure can impede the spread of complex contagions until a sizeable critical mass exists that can start to breach individuals' thresholds. They also find that for complex contagion to spread, the critical mass must also be spread throughout a network: a strongly tied group of any size cannot influence another strongly tied group with one weak tie when the contagion is complex; multiple signals are required (Figure $\underline{6}$ ). Therefore, the important factor in leading a community to success (or failure) is whether a subset of the $x$ chosen agents are connected to the same agent and although perhaps unable to influence it alone, can jointly influence it.

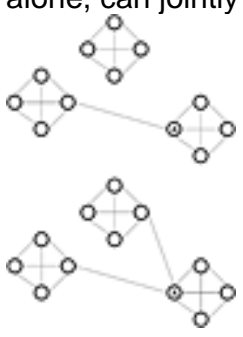

Figure 6. The upper panel shows three strongly tied groups with one weak tie between two of them allowing a simple contagion to spread to the group on the right. However, if node A needs two signals to breach its threshold, a complex contagion will not spread. A second weak tie is required (bottom panel)

Centola and Macy (2007) also report that a small world structure tends to inhibit the spread of a complex contagion when compared with a simple contagion and that a small world facilitates the spread of all contagions (simple and complex) when compared with both a fully regular model and a random model. Our results are in agreement with this second finding (our data do not allow us to comment on the first). We find that when the critical mass is able to influence at least one person, the small world structure of fnet facilitates the spread of contagion, when compared with the random configuration model: social networks are more efficient than random ones at allowing the spread of contagions of all levels of complexity, which includes ideas, disease, information and social media technologies.

\section{Limitations}

A limitation of this work is that although these conclusions do not depend on the values of the threshold parameters, they do depend on the existence of a threshold decision rule, which may appear too simplistic to capture complex human behavior. However, the threshold decision rule has been found to apply in many decisions, including as mentioned earlier, adopting certain behaviors in online social networks (Centola, 2011), 
and it is therefore not unrealistic to use it here. Another limitation is that it examines a readymade, fully formed community, where the number in the community and the network of links between them is set before the simulation is run. In reality, community membership changes over time as do the links between members. Future work should look at how this impacts on community success or failure.

One criticism that has been levelled at similar work (Watts and Dodds, 2007) is that is it based entirely on computer simulations (Watts, 2011). However, simulations are useful tools that can generate great insight (Watts, 2011).

\section{Conclusions}

This study compared a critical mass of adopters with a critical mass of those who discontinue their adoption of social media. Results showed that a critical mass of leavers can cause a community to fail and that this mass can potentially be as small as the mass needed to influence a community to succeed; although given a certain critical mass, their leaving is less likely to cause failure than their adopting is success. The influence of the critical mass is facilitated by the network structure.

All the code that was used in this study is available from the authors.

$1 . \quad 1$

In line with other studies itepNewman10, when calculating the clustering coefficient, the direction of the network edges has been ignored.

2. 2

We use here an estimated probability that is actually the observed probability calculated from the sample of 50 trials of each value of $x$.

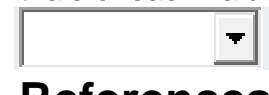

\section{References}

- Ajzen I. 1991. The theory of planned behaviour. Organizational Behavior and Human Decision 179-211.

- Ajzen I, Fishbein M. 1973. Attitudinal and normative variables as predictors of specific behaviour. Journal of Personality and Social Psychology (1): 41-57.

- Amblee N, Bui T. 2011. Harnessing the influence of social proof in online shopping: the effect of electronic word of mouth on sales of digital microproducts. International Journal of Electronic Commerce (2): 91-113.

- $\quad$ Bainbridge WS. 2010. The Warcraft Civilization. MIT Press: Cambridge Massachusetts.

- $\quad$ Barabási A, Albert R. 1999. Emergence of scaling in random networks. Science 509-512.

- $\quad$ BBC. 2011. Facebook users average 3.74 degrees of separation. http://www.bbc.co.uk/news/technology-15844230

- $\quad$ Benbasat I, Barki H. 2007. Quo vadis TAM? Journal of the Association for Information Systems (4): 211218.

- Bermudez J. 2010. Cognitive Science. Cambridge University Press: UK.

- $\quad$ Bruggeman J. 2008. Social Networks. Routledge: New York. 
- Butts C, Hunter D, Handcock M. 2007. Network: classes for relational data. http://erzuli.ss.uci.edu/R.stuff

- Centola D. 2009. Failure in complex social networks. Journal of Mathematical Sociology (1): 64-68.

- Centola D. 2010. The spread of behavior in an online social network experiment. Science (5996): 11941197.

- Centola D. 2011. An experimental study of homophily in the adoption of health behavior. Science (6060): 1269-1272.

- Centola D, Macy M. 2007. Complex contagions and the weakness of long ties. American Journal of Sociology (3): 702-734.

- Cortizo J, Carrero F, Gómez J. 2011. Introduction to the special issue: mining social media. International Journal of Electronic Commerce(3): 5.

- Csárdi G, Nepusz T. 2006. The igraph software package for complex network research. InterJournal Complex Systems.

- Davis G, Yoo M, Baker W. 2003. The small world of the American corporate elite, 1982-2001. Strategic Organization (3): 301-326.

- Easley D, Kleinberg J. 2010. Networks, Crowds and Markets. Cambridge University Press: New York.

- Granovetter M. 1973. The strength of weak ties. American Journal of Sociology 1360-1380.

- Granovetter M. 1978. Threshold models of collective behavior. American Journal of Sociology (6): 1420 1443.

- Guala F. 2002, Model Based Reasoning: Science, Technology, Values. Kluwer Academic Publishers: New York, chapter Models, simulations and experiments.

- Hirschheim R. 2007, Introduction to the special issue on 'quo vadis TAM-issues and reflections on technology acceptance research'. Journal of the Association for Information Systems (4): 203-205.

- Innis H. 1950. Empire and Communications. Claredon Press: Oxford, UK.

- Innis H. 1951. The Bias of Communications. University of Toronto Press: Oxford, UK.

- Jaccard J, Jacoby J. 2010, Theory Construction and Model-Building Skills. Guilford: New York.

- Jackson M. 2008. Social and Economic Networks. Princeton University Press.

- Java A, Song X, Finin T, Tseng B. 2007. Why we Twitter: understanding microblogging usage and communities. Proceedings of the 9th WebKDD and 1st SNA-KDD 2007 workshop on Web mining and social network analysis, ACM: New York; 56-65.

- Levy S. 2007. Twitter: is brevity the next big thing? Technical report, Newsweek. http://www.newsweek.com/2007/04/29/twitter-is-brevity-the- next-big-thing.html

- $\quad$ Liang T, Ho Y, Li Y, Turban E. 2011. What drives social commerce: the role of social support and relationship quality. International Journal of Electronic Commerce (2): 69-90.

- Liang T, Turban E. 2011. Introduction to the special issue: social commerce: a research framework for social commerce. International Journal of Electronic Commerce (2): 5-13.

- Markus M. 1987. Toward a 'critical mass' theory of interactive media. Communication Research (5): 491-511. 
- Marwell G, Oliver P. 1993. The Critical Mass in Collective Action: A Micro-Social Theory. Cambridge University Press: New York.

- Milgram S. 1967. The small world problem. Psychology Today 60-67.

- Morris S. 2000. Contagion. Review of Economic Studies 57-78.

- Newman M. 2010. Networks An Introduction. Oxford University Press: Chicago.

- Newman MEJ. 2001. Scientific collaboration networks. I. Network construction and fundamental results. Physical Review E.

- Newman MEJ, Park J. 2003. Why social networks are different from other types of networks. Physical Review E (3): 036122.

- Opuszko M, Ruhland J. 2013. Effects of the network structure on the dynamics of viral marketing. th International Conference on Wirtschaftsinformatik, Leipzig, Germany.

- Poe M. 2011. A History of Communications. Cambridge University Press.

- Prins R, Verhoef PC, Franses PH. 2009. The impact of adoption timing on new service usage and early disadoption. International Journal of Research in Marketing (4): 304-313.

- Roca C, Sanchez A, Cuesta J. 2012. Individual strategy update and emergence of cooperation in social networks. Journal of Mathematical Sociology (1): 1-21.

- Rogers E. 1995. Diffusion of Innovations 4th Edition. Free Press: New York, NY, USA.

- Schelling, T. 1973. Hockey helmets, concealed weapons and daylight saving: a study of binary choices with externalities. Journal of Conflict Resolution 381-428.

- Schelling TC. 1971. Dynamic models of segration. Journal of Mathematical Sociology 143-186.

- Simon H. 1969. The Sciences of the Artificial. MIT Press.

- Stephen A, Toubia O. 2010. Deriving value from social commerce networks. Journal of Marketing Research XLVII: 215-228.

- Tang F. 2011. Knowledge transfer in intra-organization networks. Systems Research and Behavioral Science 270-282.

Direct Link:

- Thompson R, Higgins C, Howell J. 1991. Personal computing: toward a conceptual model of utilization. MIS Quarterly (1): 124-143.

- Valente T. 1996. Social network thresholds in the diffusion of innovations. Social Networks 69-89.

- Venkatesh V, Morris M, Davis G. 2003. User acceptance of information technology: toward a unified view. MIS Quarterly (3): 425-478.

- Venkatesh V, Thong J, Chan F, Hu P, Brown S. 2011. Extending the two-stage information systems continuance model: incorporating UTAUT predictors and the role of context. Information Systems Journal doi: 10.1111/j.1365-2575.2011.00373.x

- Watts, D. 1999. Small Worlds. Princeton University Press: New York, NY, USA.

- Watts D. 2011. Everything is Obvious. Atlantic Books: New York, NY, USA.

- Watts D, Dodds P. 2007. Influentials, networks and public opinion formation. Journal of Consumer Research. 
- Watts D, Strogatz S. 1998. Collective dynamics of 'small world' networks. Nature 440-442.

- Willer R. 2007. The role of metanetworks in network evolution. Journal of Mathematical Sociology (2): 101-119.

- Winsberg E. 2010. Science in the Age of Computer Simulation. University of Chicago Press. 